\title{
Einarbeitung von Nanoclay in Polyolefine mittels Planetwalzenextruder
}

\author{
Karl-Heinz Ohrdorf', Thomas Birr ${ }^{2}$ und Helmut Flachberger ${ }^{3}$
}

II.B.O. Ingenieur-Büro für Bentonit-Technologie Dipl.-Ing. Ohrdorf, Wiesbaden, Deutschland ${ }^{2}$ ENTEX Rust \& Mitschke GmbH, Bochum, Deutschland ${ }^{3}$ Lehrstuhl für Aufbereitung und Veredlung, Department Mineral Resources Engineering, Montanuniversität Leoben, Leoben, Österreich

Eingegangen 19. Oktober 2020; angenommen 21. Oktober 2020; online publiziert 13. November 2020

Zusammenfassung: Als aktive Füllstoffe in Polymeren zum Einsatz gelangende Organoclays werden durch Kationenaustausch aus natürlichen Natrium-Bentoniten hergestellt, für die es in Europa keine industriell verwertbaren Lagerstätten gibt. Am Lehrstuhl für Aufbereitung und Veredlung der Montanuniversität Leoben konnte ein für diese Anwendung gleichwertiger Organoclay aus europäischen Kalzium-Bentoniten entwickelt werden.

Die Einarbeitung eines idealerweise vollständig exfolierten Organoclays in nichtpolare Polymere wie Polypropylen und Polyethylen gilt als sehr schwierig. Dafür sind eine schlechte Kompatibilität und die Neigung zur Re-Agglomeration der mineralischen Partikel verantwortlich.

Die Exfolierung von schichtpaketartigen Füllstoff-Agglomeraten erfordert sehr hohe Scherkräfte beim Compoundieren bei latenter Gefahr, dass dabei das Polymer geschädigt werden kann.

Erste Compoundierversuche mit einem Doppelschneckenextruder brachten nicht den gewünschten Effekt. Der hier beschriebene Planetwalzenextruder zeigte eindeutige Verbesserungen bei der Dispergierung der Agglomerate. Eine zufriedenstellende Exfolierung konnte aber auch hier nicht erzielt werden.

Es konnte nicht erklärt werden, warum sich der Füllstoff mit einem $d_{98}<5 \mu \mathrm{m}$ beim Eintrag in ein homopolymeres Polypropylen zu Aggregaten mit einem ca. 60-fach größeren Durchmesser zusammenballt, während dies beim Polyethylen nicht geschieht.

Die erwarteten Verbesserungen der mechanischen Eigenschaften wie E-Modul und Zugfestigkeit wurden auch bei höheren Füllgraden nicht erreicht.

\footnotetext{
Univ.-Prof. Dr. mont. H. Flachberger ( $\square)$

Lehrstuhl für Aufbereitung und Veredlung, Department Mineral

Resources Engineering,

Montanuniversität Leoben,

Franz-Josef-Straße 18

8700 Leoben, Österreich

helmut.flachberger@unileoben.ac.at
}

Schlüsselwörter: Organoclay aus Kalzium-Bentonit, Planetwalzenextruder

Compounds of Nanoclay in Polyolefines Using a Planetary Roller Extruder

Abstract: Organoclays as active fillers are produced from naturally occurring sodium-bentonites by cation exchange. At the Chair of Mineral Processing of the Montanuniversitaet Leoben, the conversion of European calcium-bentonites into an organoclay with comparable characteristics for such applications has been developed.

Compounding of organoclay with polyolefines is described as very difficult due to bad compatibility and the tendency of the filler to re-agglomerate. The exfoliation of the clay agglomerates requires very high shearing forces with a latent negative impact on the polymer strength.

Trials with a commonly used twin screw extruder did not show the desired efficiency. The first compounding tests with the planetary roller extruder presented in this paper result in a significantly better dispersion of the filler. However, satisfactory exfoliation of the agglomerates in polypropylene has not been achieved.

It could not be explained why a filler with a d98 of $5 \mu \mathrm{m}$ forms agglomerates almost 60 times this size when compounded with polypropylene, but no visible agglomerates in polyethylene.

The expected improvements of mechanical characteristics of the compound, such as modulus of elasticity and tensile strength, were not achieved, even at higher filling grades.

Keywords: Organoclay from calcium bentonite, Planetary roller extruder 


\section{Einführung}

In einer kaum noch überschaubaren Anzahl von Veröffentlichungen wurde vor der und um die Jahrtausendwende über den möglichen Einsatz von organophil modifiziertem Montmorillonit (dem Hauptmineral des Bentonites) als Füllstoff in Polymeren und den zu erwartenden Verbesserungen mechanischer und physikalischer Eigenschaften eines solchen Verbundwerkstoffes berichtet. Ein guter, allgemeiner Überblick über diese Thematik, ist z. B. bei Le Baron et al. beschrieben [1] und dort durch vielfältige weiterführende Veröffentlichungen unterlegt.

In der Literatur wird erwähnt, dass der Einsatz von allgemein als "Nanoclays" oder "Organoclays" bezeichneten organophil modifizierten Montmorilloniten in allen industrieüblichen Polymeren möglich ist. Bahnbrechende Ergebnisse wurden nach dem Toyota-Verfahren mit Nylon-6, einem Polyamid, erzielt. Die Einbringung von Nanoclays in Polyolefine, wie z. B. Polypropylen, gilt jedoch als herausfordernd schwierig, da die Polarität der Polyolefine nicht gut mit der der Organoclays zusammenpasst.

Bei Zakaria [2] wurde aber auch festgestellt, dass sich die hohen Erwartungen an die angestrebte Verbesserung der Materialeigenschaften oft nicht erfüllten. Die Ursache dafür konnte in der Art der Verteilung der Nanoclay-Partikel im Polymer ausgemacht werden:

- als Agglomerate mit dem geringsten Aspektverhältnis, die unter dem Blickpunkt einer Festigkeitssteigerung eher wie Fehlstellen in der Matrix fungieren,

- als Aggregate, die durch Interkalation von Polymermolekülen in die Zwischenschichten aufgeweitet werden und so das Aspektverhältnis sowie die spezifische äußere Oberfläche und die Haftkräfte an dieser vergrößern, und

- die als Delamellierung oder Exfolierung bezeichnete vollständige Aufspaltung mit dem jeweils maximalen Aspektverhältnis bzw. der maximalen spezifischen äußeren Oberfläche.

Vollständig in Einzellamellen exfolierte Schichtpakete in die Matrix einzubringen, ist sicherlich der anzustrebende, ideale Zustand, industriell-technisch aber wohl nicht erreichbar. Andererseits kann die immer wieder positiv bewertete Interkalation der Polymermoleküle in die Zwischenschichten ebenfalls nicht zu einer idealen Matrix aus Polymer und Füllstoff führen. Die interkalierten Polymermoleküle weiten zwar den Schichtabstand der Lamellen im Agglomerat auf, was aber offensichtlich nicht ausreicht, um bei den beschriebenen Füllgraden von $1 \ldots 5 \mathrm{~m} \%$ Nanoclay eine homogene Verteilung in der Gesamtmatrix zu erzielen, die für die Maximierung der mechanischen Eigenschaften erforderlich ist. Eigene Untersuchungen zeigen, dass bei Polypropylen überhaupt keine Interkalation zu detektieren war und dass das Eindringen in die Zerklüftungen der aus sehr unregelmäßig geformten Lamellen bestehenden Agglomerate als Fehlinterpretation einer Interkalation angesehen werden muss. Interkalation beginnt mit der Keilporen-artigen Aufweitung der Randzonen der Aggregate/Schichtpakete. Es bedarf zumindest des eindeutigen Erkennens dieser Aufweitungen, wenn man davon ausgeht, dass die sehr kur-

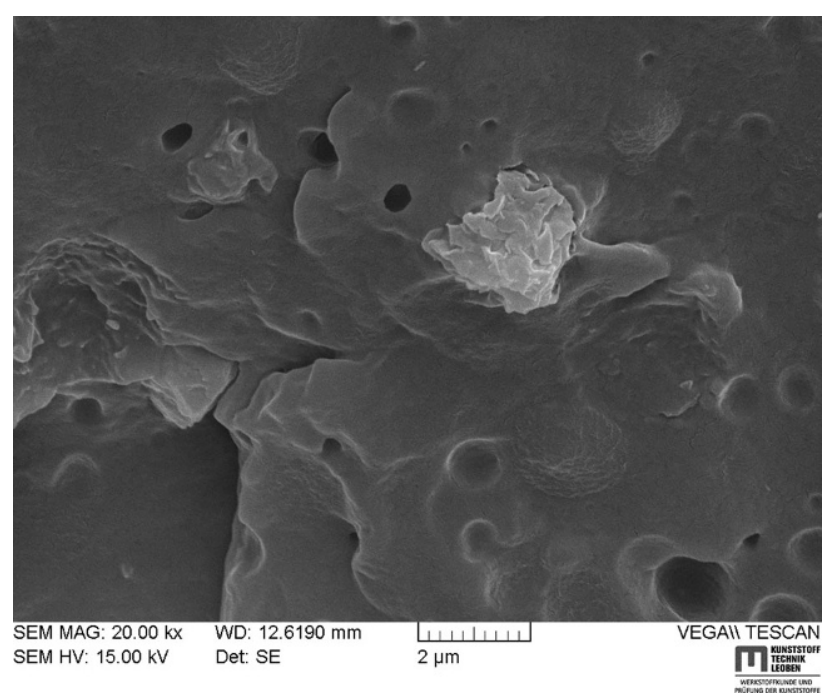

Abb. 1: Montmorillonit-Schichtpaket in Polypropylen nach Einarbeitung mit einem Kneter. Quelle: E. Krischey, Lehrstuhl für Aufbereitung und Veredlung, Montanuniversität Leoben

zen Verweilzeiten beim Compoundieren ohnehin keine Vollständigkeit einer Interkalation gewährleisten können.

Abb. 1 und 2 zeigen ein mit einem Kneter in Polypropylen eingearbeitetes Montmorillonit-Schichtpaket. Klar zu erkennen ist, dass weder eine Exfolierung noch eine Interkalation stattfand. Abb. 3 zeigt ein mit einem Kneter in Polypropylen eingearbeitetes Montmorillonit-Schichtpaket nach einem Zugversuch. Auch hier ist keine Interkalation zu erkennen.

Obwohl es mehrere Möglichkeiten des Einbringens des Füllstoffes in ein Polymer gibt, hat sich die Schmelzcompoundierung als technischer Standard durchgesetzt. Die Verwendung von Twin-Extrudern wird präferiert. Hier kann besonders auf die Untersuchungen von A. Witschnigg des Department Kunststofftechnik der Montanuniversität verwiesen werden [3]. Von einem der führenden NanoclayHersteller wird der Einsatz von gleichlaufenden Doppel-

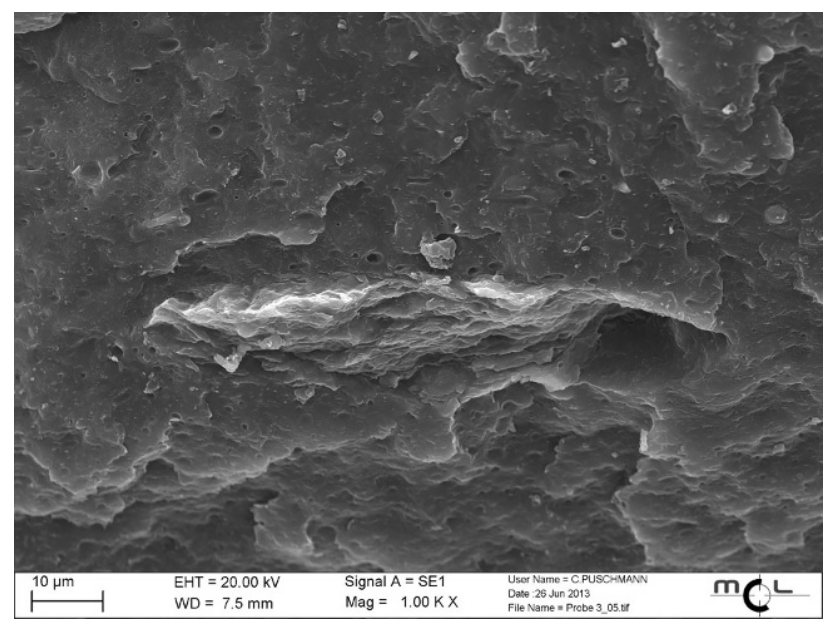

Abb. 2: Montmorillonit-Schichtpaket in Polypropylen nach Einarbeitung mit einem Kneter. Quelle: E. Krischey, Lehrstuhl für Aufbereitung und Veredlung, Montanuniversität Leoben 


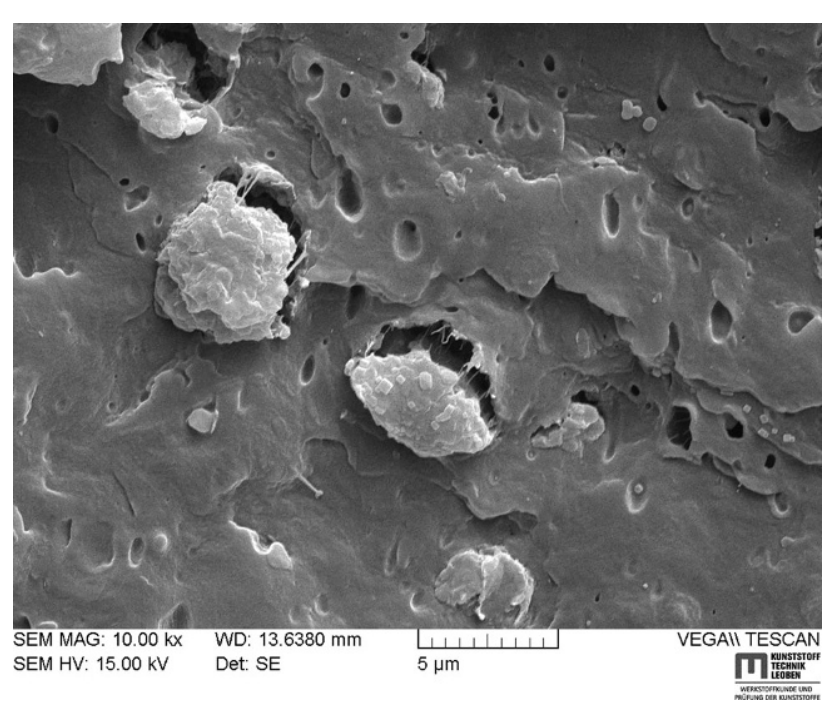

Abb. 3: Montmorillonit-Schichtpaket in Polypropylen nach Zugversuch (nach Einarbeitung mit einem Kneter). Quelle: E. Krischey, Lehrstuhl für Aufbereitung und Veredlung, Montanuniversität Leoben

schnecken-Extrudern sowie eines Buss Co-Kneters empfohlen [4].

Eigene Untersuchungen zeigten jedoch auf, dass mit dem Twin-Extruder zwar eine recht gute Verteilung der Partikel, nicht jedoch eine Exfolierung der Agglomerate erzielt werden konnte (Abb. 4).

Beim Compoundieren kommt es im Wesentlichen auf folgende Faktoren an:

- Die Einbringung von Scherkräften zur Überwindung der elektrostatischen Kräfte, die die Montmorillonit-Lamellen in Aggregaten zusammenhalten,

- die Einstellung einer Viskosität, die zwar den Energieeintrag zur Exfolierung gewährleistet, aber nicht zur Schädigung des Polymers führt, und

- die Verweilzeit des Mischens.

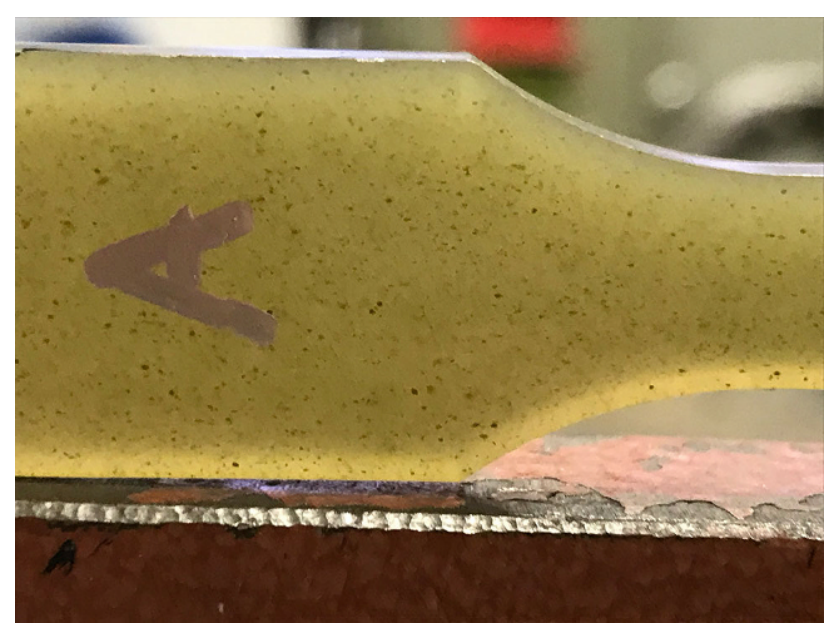

Abb. 4: Füllgrad von $5 \mathrm{~m} \%$ Nanoclay der eigenen Entwicklung in Polypropylen Bormed ${ }^{\mathrm{TM}}$ DM55pharm, compoundiert am Lehrstuhl für Spritzgießen von Kunststoffen der Montanuniversität Leoben. Quelle: A. Neunhäuserer/N. Horn, Lehrstuhl für Spritzgießen der Kunststoffe/Lehrstuh für Aufbereitung und Veredlung, Montanuniversität Leoben
In der Zwischenzeit hat sich die anfängliche Euphorie gelegt. Vielleicht lag es herstellungsbedingt am hohen Preis dieser Füllstoffe, an der für europäische Hersteller nicht immer gesicherten Verfügbarkeit hochwertiger, zumeist nordamerikanischer Rohbentonite (natürliche Natrium-Bentonite), an der Prozesstechnik, die eigentlich für die Herstellung solcher Art modifizierter Bentonite zur Anwendung als rheologische Stellmittel in Öl-basierten Lacken und Farben entwickelt wurde, oder eben an der als wesentlichen letzten Baustein zu betrachtenden Einarbeitung des Füllstoffes in das Polymer.

Vor allem zwei Haupt-Argumente, die für den Einsatz dieses Füllstoffes sprächen, sind jedoch immer noch überzeugend:

- das hohe Aspektverhältnis der Montmorillonit-Lamellen zur Verbesserung mechanischer und physikalischer Eigenschaften des Polymers, und

- seine Flammen- und Abtropf-hemmende Wirkung z.B. bei Kabelummantelungen

Vor nunmehr über zehn Jahren wurden die Entwicklung eines solchen Füllstoffes aus europäischen Rohbentoniten durch den Lehrstuhl für Aufbereitung und Veredlung der Montanuniversität Leoben in Kooperation mit der Firma I.B.O. Ingenieur-Büro für Bentonit-Technologie Dipl.-Ing. Ohrdorf, Wiesbaden, sowie den drei Lehrstühlen für Chemie der Kunststoffe, Kunststoffverarbeitung und Spritzgießen von Kunststoffen der Montanuniversität Leoben als wissenschaftliche Herausforderung angenommen. Sie wurde noch dadurch erweitert und erschwert, sich für das gegenüber Polyamiden preiswertere, aber eben auch als schwierigerer Partner angesehene Polypropylen als Massenkunststoff entschieden zu haben.

Im Rahmen einer Dissertation und Publikation an gleicher Stelle [5], wurden als erstes Ziel genannt und begründet, wobei beim Rohgut immer von Bentonit gesprochen wird:

- Erlangen einer Unabhängigkeit vor allem von nordamerikanischen, aber auch asiatischen Natrium-Bentonit-Lagerstätten, wie auch von deren Produkten (blends).

- Im europäischen Raum, einer politisch und wirtschaftlich stabilen Region, befinden sich rund um das Mittelmeer wie auch in Deutschland ausreichende und für die vorgesehene Anwendung geeignete Bentonit-Vorkommen, und zwar mit Reserven, die die Versorgung auf Jahrzehnte absichern.

- Bessere Verfügbarkeit und verringerte Transportkosten

An gleicher Stelle wurde aber auch formuliert, dass es einer besonderen verfahrenstechnischen Entwicklung bedarf, um die Nachteile der europäischen Kalzium-Bentonite, die bisher für die organophile Modifizierung als wenig bis ungeeignet angesehen wurden, auszugleichen.

Dabei musste im Laufe der Entwicklung folgender Gedankenkette nachgegangen werden: 


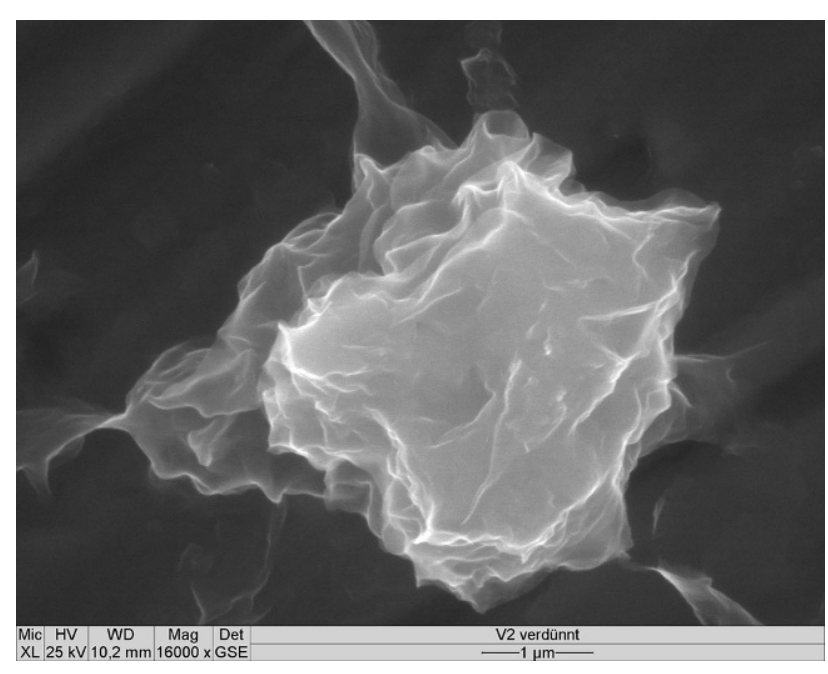

Abb. 5: Agglomerierte Montmorillonit-Lamellen. Quelle: K.-H. Ohrdorf, I.B.O. Ingenieur-Büro für Bentonit-Technologie Dipl.-Ing. Ohrdorf

1. Die Verbesserung der mechanischen Werkstoffeigenschaften beruht auf dem Aspektverhältnis des Füllstoffes und der wirksamen äußeren Oberfläche der Montmorillonit-Lamellen im Haftverbund mit dem Polymer.

2. Das in Schichtpaketen (Abb. 5) von bis zu 500 Einzellamellen vorliegende Mineral muss also so weit wie es technisch machbar ist, in seine Einzellamellen (Abb. 6) von ca. 1-2 nm Dicke exfoliert werden. Theoretisch sind dabei Aspektverhältnis-Werte von bis zu 2000 möglich.

3. Auf Grund der elektrostatischen Wechselwirkungen ist eine solche Exfolierung von Kalzium-Montmorilloniten nicht möglich; die Überführung in Natrium-Montmorillonite ist daher ein erster notwendiger Schritt.

4. Die Überführung in Natrium-Bentonit, auch bekannt als „alkalische Aktivierung , reicht aber für eine effektive Exfolierung allein nicht aus, sondern es müssen dafür zusätzlich hohe Scherkräfte aufgewendet werden.

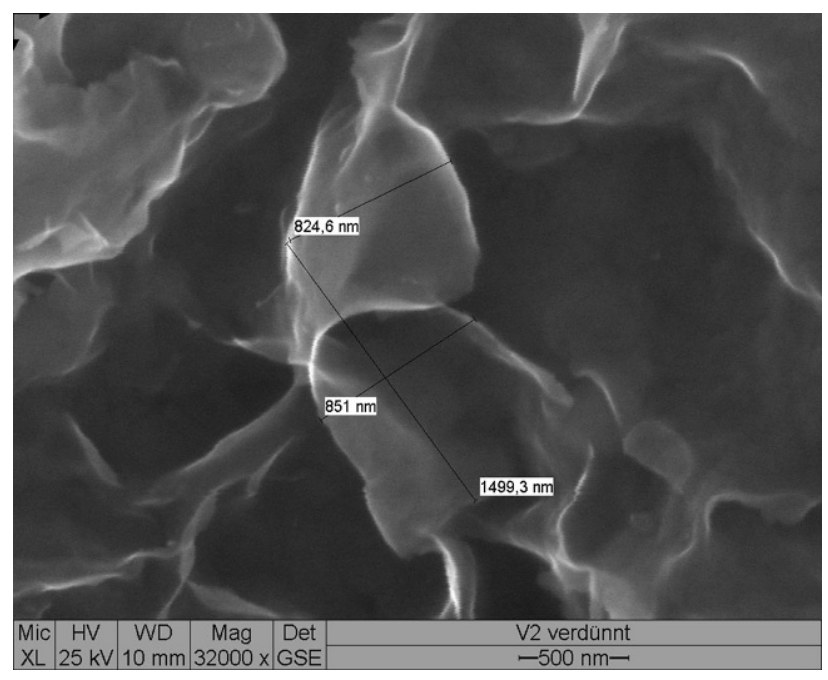

Abb. 6: Vereinzelte Montmorillonit-Lamellen. Quelle:K.-H. Ohrdorf, I.B.O. Ingenieur-Büro für Bentonit-Technologie Dipl.-Ing. Ohrdorf
5. Die hydrophilen Montmorillonit-Lamellen müssen zum Polymer kompatibel gemacht werden. Das geschieht in der Praxis über den Austausch der Natrium-Ionen durch quaternäre Ammonium-Ionen. Da aber auf Grund unterschiedlicher atomarer Strukturen in dem dioktaedrisch kristallisierenden Montmorillonit nicht an allen Stellen gleichermaßen ein organisches Molekül angedockt werden kann, bleiben hydrophile Bereich übrig.

6. Die Verfahrensstufe der Exfolierung kann nur in der Suspensionsphase erfolgen. Um den Füllstoff verarbeitungsfähig zu machen, muss er jedoch auf eine sehr geringe Restfeuchte ( $<10 \mathrm{~m} \%$ ) getrocknet werden. Dabei wird der Zustand der Exfolierung temporär aufgehoben. Es entstehen erneut Agglomerate.

7. Beim Compoundieren müssen also die Scherkräfte aufgewendet werden, die notwendig sind, das Trockengut zu de-agglomerieren und zugleich so zu mischen, dass der Füllstoff homogen im Polymer zu einem Verbundwerkstoff verteilt vorliegt. Eigene Untersuchungen kommen zu dem Schluss, dass es in der industriellen Praxis wohl nicht möglich sein wird, eine vollständige Vereinzelung in Einzellamellen zu erzielen, sondern dass es sich überwiegend um dünnste Schichtpakete einiger weniger Lamellen handeln wird. Ein Aspektverhältnis von ca. 300 .. 500 wird dennoch als erreichbar angesehen, denn die pseudo-hexagonalen Montmorillonit-Lamellen sind zudem in ihrer längsten Ausrichtung ungleichförmig ausgeprägt.

8. Welche Einarbeitungstechnologie soll zum Einsatz kommen, nachdem mit dem Doppelschnecken-Extruder die Exfolierung nicht erreicht wurde?

Wie wurden die genannten Schritte abgearbeitet und was wurde erreicht?

1. Für die Versuche wurde ein Rohbentonit einer Lagerstätte auf Sardinien ausgewählt, der die genannten Kriterien erfüllt.

2. Die Berechnung der Zugabe der Reaktanden für die alkalische Aktivierung und der darauf beruhenden organophilen Modifizierung erfolgte nach der I.B.O.-Methode [6].

3. Als herausragendes Ergebnis kann die Entwicklung eines Suspensionsverfahrens angesehen werden, bei dem in einem einzigen Reaktionsgefäß, aber zeitlich nacheinander gestaffelt und unter jeweils angepassten Prozessparametern, alle drei Verfahrensstufen: 1) Umwandlung des Kalzium-Bentonites in Natrium-Bentonit, 2) Exfolierung und 3) organophile Modifizierung ermöglicht [7].

4. Aus labortechnischen Gründen war nur ein Eindampfen der Suspension im üblichen Trockenschrank möglich.

5. Die anschließend notwendige De-Agglomerierung erfolgte auf einer Spiralstrahlmühle der Firma PMT Jetmill GmbH, Kammern, Österreich. Auf Grund der hohen Reinheit des Rohbentonites (ca. 92\% MontmorillonitGehalt, absolut vergleichbar mit dem auf dem Markt befindlichen Produkt "Cloisite“ aus ausgereinigtem amerikanischen Natrium-Bentonit) wurde im Rahmen der vorliegenden Arbeit auf die ursprünglich ange- 
dachte Ausreinigung verzichtet. Gleichzeitig wurden dabei auch die wenigen enthaltenen Begleitminerale zerkleinert. Die Feinheit des für die Compoundierung erhaltenen Füllstoffes wurde mit $d_{98}=4,98 \mu$ gemessen.

6. Für die ersten Versuche zur Einarbeitung wurde vom Lehrstuhl für Kunststoffverarbeitung ein Block Copolymer Polypropylen BB412E von Borealis ausgewählt, danach wurde vom Lehrstuhl für Spritzgießen von Kunststoffen auf ein Homopolymer Bormed ${ }^{\mathrm{TM}}$ DM55pharm umgestellt. Alle Einarbeitungsversuche mit dem am Lehrstuhl für Spritzgießen von Kunststoffen verfügbaren Doppelschnecken-Extruder zeigten, dass zwar eine recht gute Verteilung gelang, die Bedingungen für eine Exfolierung aber nicht geschaffen werden konnten (Abb. 4).

7. Die Suche nach einer alternativen Extrudertechnik führte zum Planetwalzenextruder, dessen Wirkungsweise und Unterschiede zum Doppelschneckenextruder nachfolgend beschrieben werden. Am 25.08.2020 wurden nach Beratungen mit einem Masterbatch-Hersteller (deutsche Vertretung der Firma JM Polymers) und der Vermittlung durch die Firma BKF Maschinentechnik GmbH, Bochum, Versuche auf einem Technikums-Planetwalzenextruder der Firma ENTEX Rust \& Mitschke GmbH, Bochum, gefahren.

\section{Compoundierversuche mit dem Planet- walzenextruder}

\subsection{Funktionsbeschreibung des Planetwalzenex- truders}

Der Planetwalzenextruder ist ein System, das neben einer Vielzahl anderer Einsatzgebiete auch für die Herstellung von Polymercompounds eingesetzt wird. Aufgrund seines Funktionsprinzips findet man diesen Maschinentyp häufig in der Verarbeitung empfindlicher und/oder hochgefüllter Compounds, da der mechanische Energieeintrag in das Material gezielt auf die jeweilige Anwendung abgestimmt werden kann. Dadurch kann vermieden werden, dass Materialien trotz intensiver Dispergierung und Durchmischung durch übermäßige Scher- oder Temperaturbelastung geschädigt werden.

Gegenüber Doppelschneckenextrudern weist der Planetwalzenextruder ein grundlegend anderes Funktionsprinzip auf. Dieses entspricht einem um $45^{\circ}$ schrägverzahnten Planetengetriebe, das über das Sonnenrad angetrieben wird. Da die Verzahnungen der Elemente ineinandergreifen, rollen die Planetspindeln auf der Zentralwelle und dem Walzenzylinder ab. Das Extrudat wird dabei erfasst und an die Wandungen gepresst. Hier wird es verdrängt und teilweise überrollt und erfährt dabei Strömungs- und Belastungsmechanismen, die mit denen in einem Walzwerksspalt vergleichbar sind. Der lokal auftretende, mechanische Energieeintrag bei einem einzelnen Überrollvorgang ist vergleichsweise gering. Durch die hohe Anzahl von Walzspalten in einem Planetwalzenextruder werden diese Effekte jedoch auf engstem Raum vielfach wieder- holt, worauf die hohe Misch- und Dispergierleistung des Systems zurückzuführen ist.

Bei den Compoundierversuchen wurde zwei forschungsrelevanten Fragestellungen nachgegangen: Kann mit der Schmelzcompoundierung überhaupt eine Exfolierung der Füllstoffagglomerate gelingen und, wenn ja, gibt es ein Maximum des Füllgrades, bis zu dem die mechanischen Eigenschaften des Verbundwerkstoffes ansteigen, um danach wieder abzufallen?

Es wurden dafür die Effekte der Extruderdrehzahl, des Füllgrades mittels Werkzeugwiderstand und zweier unterschiedlicher Dosierszenarien auf das Ergebnis untersucht. Der Gesamtmassedurchsatz wird in allen Einstellungen konstant gehalten.

Ebenso sollten zu Vergleichszwecken zwei verschiedene Polyolefintypen, Polypropylen und Polyethylen, zum Einsatz kommen.

\subsection{Versuchsaufbau}

In Abb. 7 ist der Versuchsaufbau schematisch dargestellt.

Der Maschinenaufbau umfasst einen Extruder mit einem Einschnecken-Füllteil und drei Walzenzylindern, die jeweils mit einer Doppelschnecken-Seitenbeschickung ausgestattet sind. An diesen werden zwei gravimetrisch arbeitende Dosierwerke positioniert, sodass Mineralstoff und Polymer unabhängig voneinander in variablem Verhältnis und an verschieden Positionen zugegeben werden können. An Walzenzylinder drei wird eine Vakuumentgasung des Compounds vor dem Austritt vorgenommen, um einen blasenfreien Strang zu gewährleisten. Der Extruder ist mit einer Strangdüse ausgestattet, um die austretende Masse in einem Wasserbad abzukühlen und zu granulieren (Abb. 8).

\subsection{Versuchsdurchführung}

In den ersten Versuchseinstellungen mit einem Füllgrad von $5 \mathrm{~m} \%$ wurde das Polymer Polypropylen Eltex ${ }^{\circledR} P$ HY001P in die Seitenbeschickung an Walzenzylinder 1 dosiert, der organophil modifizierte Bentonit in jenen an Walzenzylinder 2. Ziel war zunächst, das Polymer vollständig aufzuschmelzen und den Füllstoff in die Schmelze einzumischen. Der Extruder war mit einer Strangdüse mit drei Bohrungen von jeweils $3 \mathrm{~mm}$ Durchmesser ausgestattet.

Als erster Parameter wurde der Einfluss zunehmender Drehzahl zwischen 80 und $280 \mathrm{~min}^{-1}$ untersucht. Der spezifische mechanische Energieeintrag stieg im Zuge dieser Veränderung von 0,13 auf $0,4 \mathrm{kWh} / \mathrm{kg}$, die spezifische thermische Leistung der Maschine verringerte sich analog dazu von 0,75 auf $0,3 \mathrm{kWh} / \mathrm{kg}$, die Masseaustrittstemperatur stieg um ca. $7^{\circ} \mathrm{C}$.

Ein positiver, aber eher geringer Effekt auf die Dispergiergüte war zu erkennen. In den Materialproben war bei niedrigen Drehzahlen eine hohe Anzahl an Füllstoff-Stippen enthalten, die mit bloßem Auge sichtbar waren. Sowohl die Anzahl als auch der Durchmesser der Stippen verringerten sich mit zunehmender Drehzahl, ein stippenfreies Material 
Hier steht eine Anzeige.

글 
Hier steht eine Anzeige.

글 


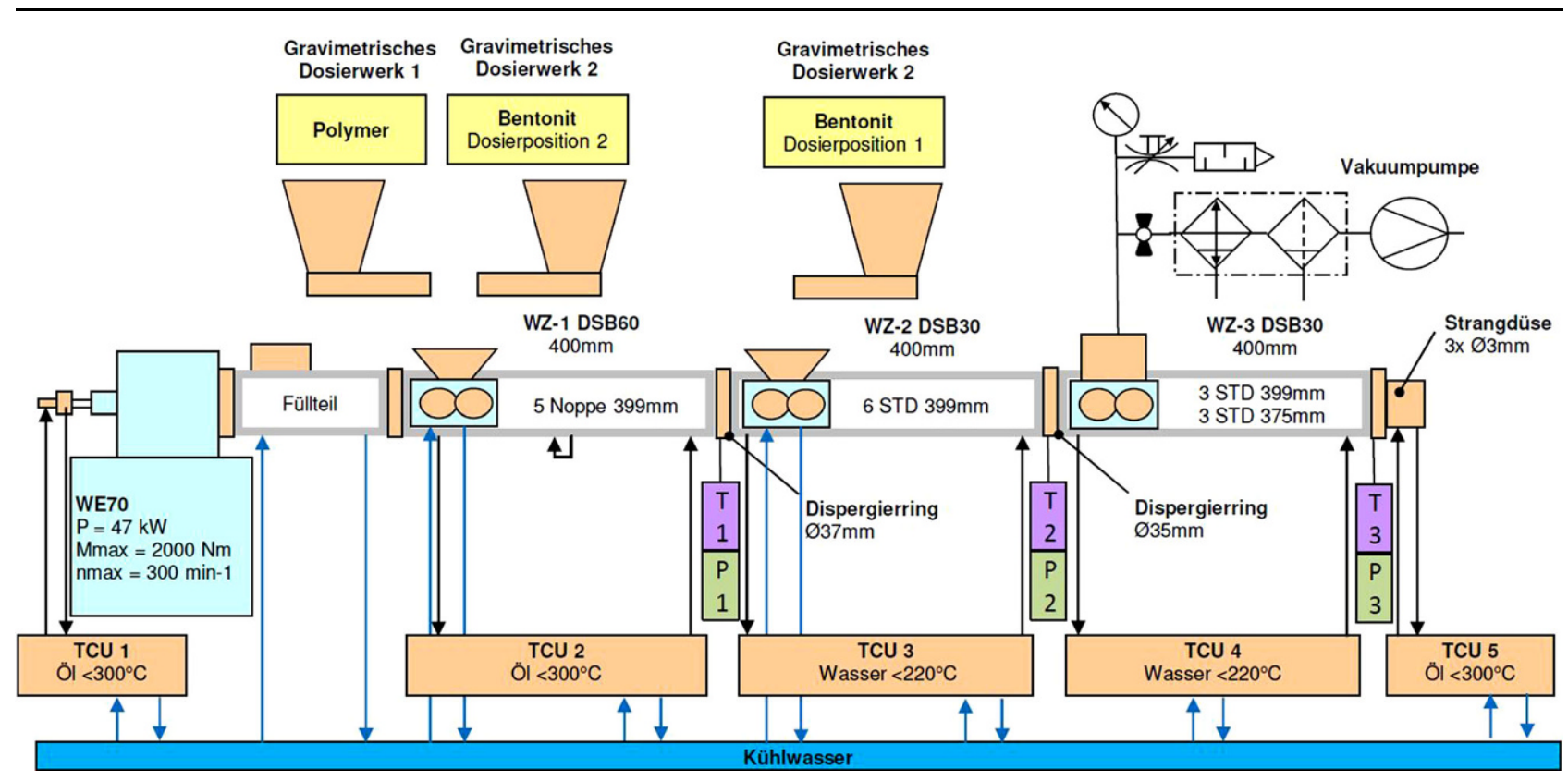

Abb. 7: Schematische Darstellung des Versuchsaufbaus. Quelle: Schematische Darstellung des Versuchsaufbaus, Freigabe durch Fa. ENTEX GmbH, Bochum

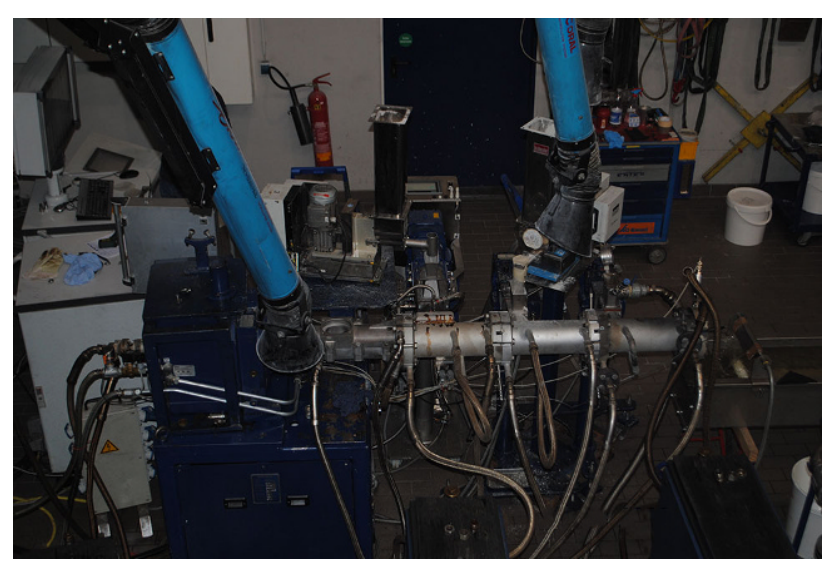

Abb. 8: Versuchsaufbau des Planetwalzenextruders. Quelle: Versuchsaufbau der Versuche vom 25.08.2020, Freigabe durch Fa. ENTEX $\mathrm{GmbH}$

konnte durch die alleinige Veränderung der Drehzahl nicht erzielt werden.

Um eine längere Verweilzeit und einen höheren mechanischen Energieeintrag in das Compound zu erzielen, wurde das Extrusionswerkzeug ausgetauscht. Das nun eingesetzte Werkzeug wies nur eine Bohrung mit einem Durchmesser von $3 \mathrm{~mm}$ auf.

Die beste Einstellung aus der vorherigen Reihe wurde wiederholt, das Ergebnis war deutlich besser - es sind nur noch vereinzelte Stippen im Material mit bloßem Auge sichtbar. Eine Betrachtung der Proben unter dem Mikroskop zeigte eine gute Einmischung des Füllstoffes, wobei sich zumindest eine gleichmäßige Verteilung ähnlich großer Agglomerate über die ganze Probe erkennen lässt. Der spezifische mechanische Energieeintrag lag in dieser Einstellung nun bei $0,43 \mathrm{kWh} / \mathrm{kg}$ bei unveränderter Austrittstemperatur.
Die Dosierposition des Mineralstoffes wurde geändert, beide Stoffe wurden nun über die Seitenbeschickung an Walzenzylinder 1 zugeführt. Durch diese Veränderung sollte einerseits eine höhere Verweilzeit des Mineralstoffes im Extruder und andererseits eine zusätzliche Feststoffreibung mit dem zunächst noch unaufgeschmolzenen Polymergries mit einer Partikelgröße $<800 \mu \mathrm{m}$ erzielt werden.

Danach wurden vergleichbare Einstellungen zu der vorherigen Dosierkonstellation erprobt und Füllstoffanteile von 5 bis $30 \mathrm{~m} \%$ zudosiert.

\section{Ergebnisse}

\subsection{Optische Beurteilung der Ergebnisse}

\subsubsection{Untersuchungen an den mit Polypropylen hergestellten Compounds}

Die optische Beurteilung der Proben erfolgte im Durchlichtmikroskop. In der vergleichenden Gegenüberstellung ist eine deutliche Verbesserung der Dispergiergüte der mittels Doppelschneckenextruder (Abb. 9) und Planetwalzenextruder (Abb. 10) erzeugten Compounds an der Verkleinerung der Agglomerate erkennbar. Mit höherem Füllgrad nehmen die Durchmesser der Agglomerate wieder deutlich zu (Abb. 11).

\subsubsection{Untersuchungen an den mit Polyethylen hergestellten Compounds}

Die zuvor gefahrenen Einstellungen zwischen 5 und $15 \mathrm{~m} \%$ Füllstoffanteil wurden nun mit dem zweiten Polymer, Polyethylen Eltex $\AA^{\circledR}$ A3180PN1852, reproduziert. 


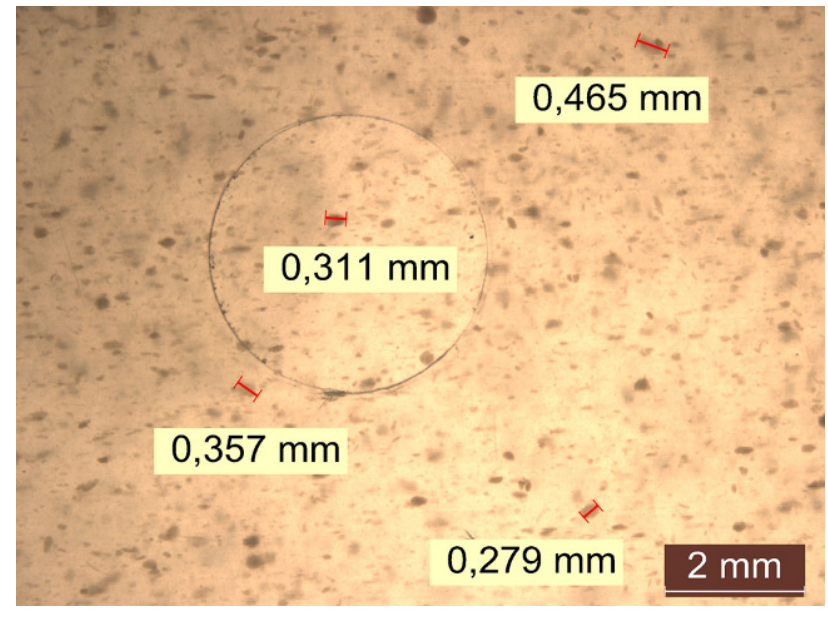

Abb. 9: Mittels Doppelschnecken-Extruder hergestellter CompoundFüllgrad $5 \mathrm{~m} \%$ organophil modifizierter Bentonit in Polypropylen. Quelle: Dr. W. Ofner, Lehrstuhl für Aufbereitung und Veredlung, Montanuniversität Leoben

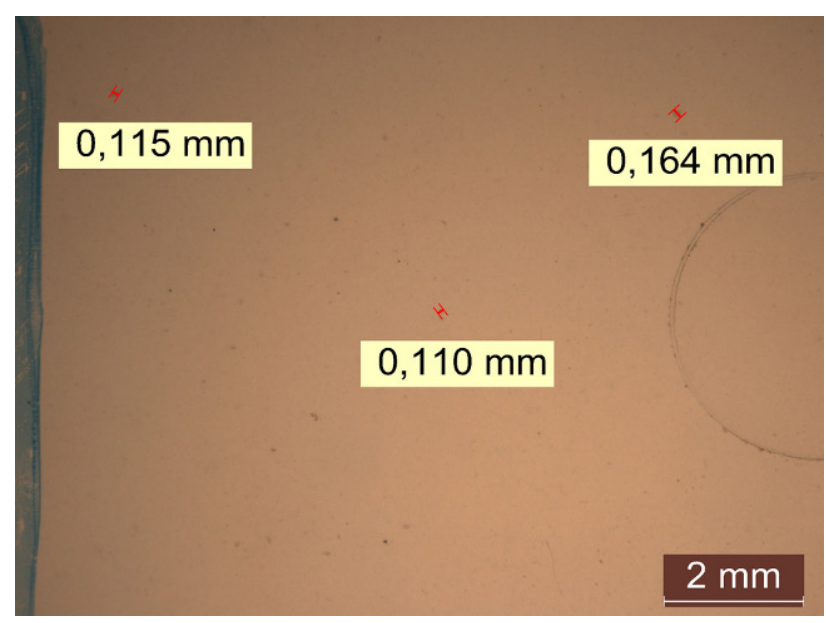

Abb. 10: Mittels Planetwalzenextruder hergestellter Compound-Füllgrad $5 \mathrm{~m} \%$ organophil modifizierter Bentonit in Polypropylen. Quelle: $\mathrm{Dr}$. W. Öfner, Lehrstuhl für Aufbereitung und Veredlung, Montanuniversität Leoben

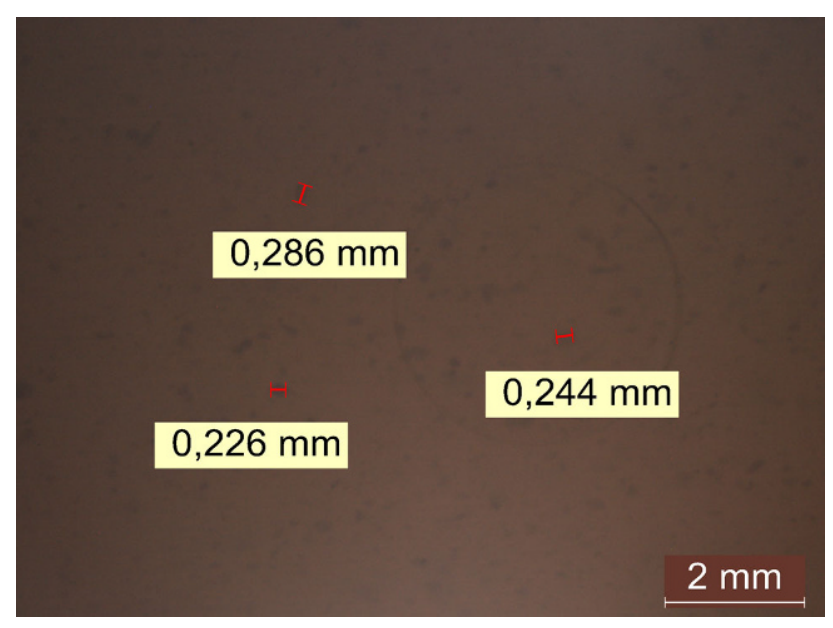

Abb. 11: Mittels Planetwalzenextruder hergestellter Compound-10 m\% organophil modifizierter Bentonit in Polypropylen. Quelle: Dr. W. Öfner, Lehrstuhl für Aufbereitung und Veredlung, Montanuniversität Leoben

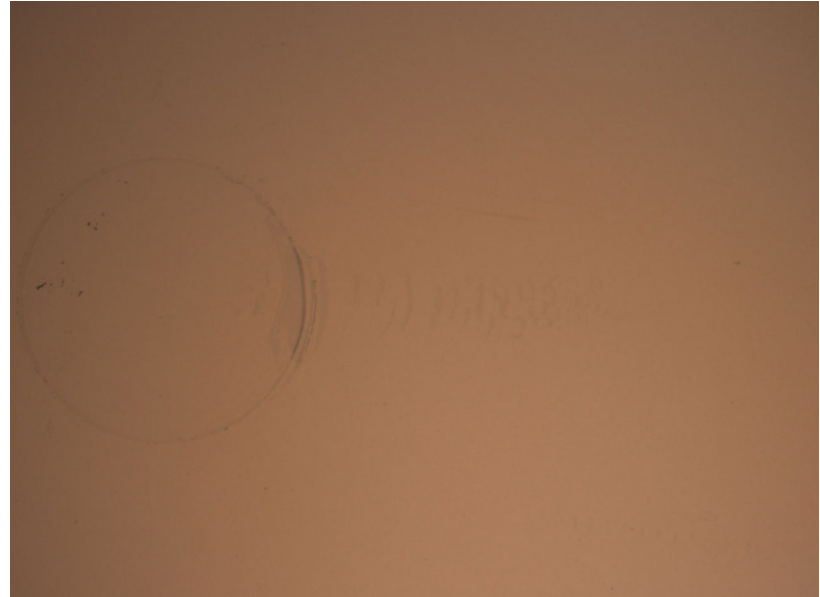

Abb. 12: Mittels Planetwalzenextruder hergestellter Compound-Füllgrad $5 \mathrm{~m} \%$ organophil modifizierter Bentonit in Polyethylen. Quelle: Dr. W. Öfner, Lehrstuhl für Aufbereitung und Veredlung, Montanuniversität Leoben

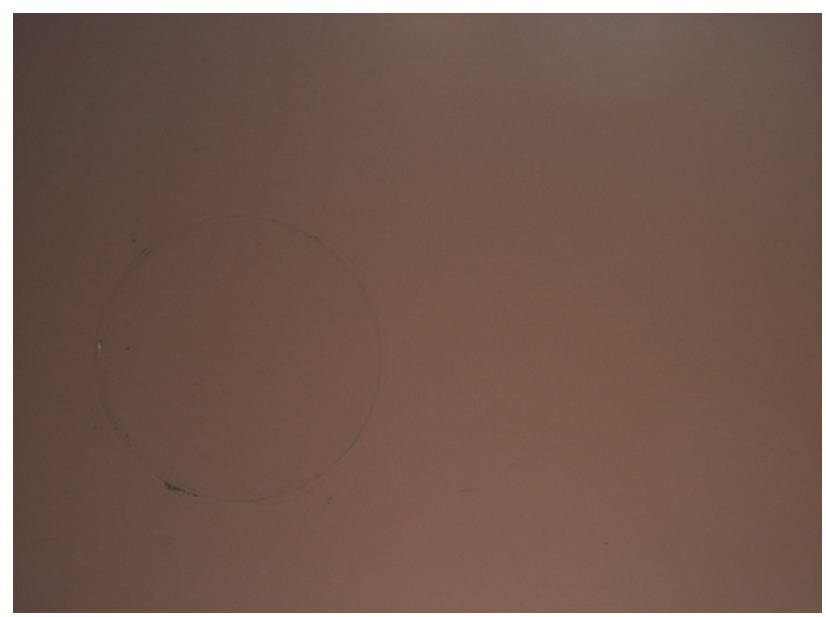

Abb. 13: Mittels Planetwalzenextruder hergestellter Compound-Füllgrad $10 \mathrm{~m} \%$ organophil modifizierter Bentonit in Polyethylen. Quelle: Dr. W. Öfner, Lehrstuhl für Aufbereitung und Veredlung, Montanuniversität Leoben

Bei der mikroskopischen Betrachtung zeigt sich ein völlig anderes Bild. Es sind nun keinerlei Agglomerate mehr erkennbar, die Presslinge zeigen lediglich eine homogene Einfärbung durch den Füllstoff (Abb. 12 und 13).

\subsubsection{Fazit}

Die Untersuchungen mit einem Durchlichtmikroskop können keine TEM- oder REM-Aufnahmen ersetzen. Wie aber aus einem Füllstoff mit $d_{98}<5 \mu \mathrm{m}$ im Polypropylen Eltex ${ }^{\circledR} P$ HY001P Agglomerate von bis zu $286 \mu \mathrm{m}$ entstehen konnten, diese aber bei Polyethylen Eltex ${ }^{\circledR}$ A3180PN1852, unter identischen Bedingungen compoundiert, nicht auftreten, kann zum gegenwärtigen Zeitpunkt der Untersuchungen nicht erklärt werden. Es bleibt daher auch weiterhin ungeklärt, ob zumindest beim Polyethylen eine Exfolierung der Agglomerate vorliegt oder nicht. 
Durch die Verarbeitung der Rezepturen auf einem Planetwalzenextruder konnte eine gute bis sehr gute Dispergierung des Füllstoffes erreicht werden, was die Grundlage für eine Exfolierung des Montmorillonits sein könnte. Die Durchmesser der größten detektierten Agglomerate haben sich bei gleichem Füllgrad von $5 \mathrm{~m} \%$ um das 2,8-fache verringert. Dies gelang in der Versuchsreihe bereits auf einem Standard-Maschinensetup, das noch nicht für diese Rezeptur optimiert wurde. Aufgrund der vielversprechenden Ergebnisse mit den Polyethylen-Rezepturen wird erwartet, dass durch eine Anpassung der Maschinenkonfiguration eine weitere Verbesserung erzielt und auch die Übertragung der Erkenntnisse auf weitere Polymertypen erfolgen kann.

Es konnte eine klare Korrelation des mechanischen Energieeintrages mit der Mischgüte des Compounds festgestellt werden, was den theoretischen Vorannahmen entspricht.

Vorteilhaft zeigte sich in diesem Zusammenhang, dass die Massetemperaturen trotz steigendem mechanischem Leistungseintrag nur unwesentlich zunehmen, da das Temperiersystem des Extruders einen entsprechenden Leistungsausgleich im Gesamtenergieeintrag gewährleistet. Die Betrachtung der Anlagendaten diesbezüglich zeigen, dass selbst bei den höchsten in der Versuchsreihe erreichten spezifischen Leistungen noch immer Wärmeenergie über die Temperierung zugeführt wurde - eine thermische Schädigung des Polymers konnte bei keiner der Proben festgestellt werden. Dies lässt darauf schließen, dass noch großes Potenzial für eine Prozessoptimierung hin zu noch höherem mechanischem Energieeintrag zur Verbesserung der Mischgüte zur Verfügung steht.
Die Erkenntnisse aus der Versuchsreihe lassen durch eine gute Vergleichbarkeit zu ähnlichen Anwendungen auch darauf schließen, dass die Skalierung des Prozesses auf einen Produktionsmaßstab unter Einsatz eines entsprechend größeren Planetwalzenextruders machbar ist. Dieser entspräche in seiner grundlegenden Konfiguration dem Versuchsaufbau, wobei hier noch eine detaillierte Untersuchung der beiden Dosierkonfigurationen im Zuge einer Prozessoptimierung durchgeführt werden soll.

Die entscheidende Frage nach der Verbesserung der mechanischen Eigenschaften des Verbundwerkstoffes gegenüber dem ungefüllten Polymer sollte zunächst durch Zugversuche nach DIN EN ISO 527-2 (06-2012) festgestellt werden. Dazu wurden Probekörper nach DIN EN ISO 3167 (201411) Typ A im Spritzgießverfahren hergestellt.

\subsection{Ergebnisse der Festigkeitsuntersuchungen}

\subsubsection{Untersuchungen des Lehrstuhls für Spritzgießen von Kunststoffen}

Vorausgegangene Untersuchungen des Lehrstuhls für Spritzgießen von Kunststoffen zeigten die in Abb. 14 ausgewiesenen Ergebnisse. Bei den untersuchten Compounds wurde Polypropylen mit der Produktbezeichnung PP BormedTM DM55pharm in unterschiedlichen Anteilen (5 und $15 \%)$ mit und ohne den Compatibilizer mit der Produktbezeichnung Scona TPPP 2112 (5\%) eingesetzt. Es zeigte sich, dass aufgrund des geringen Exfolierungsgrades weder mit noch ohne Compatibilizer - eine wesentliche

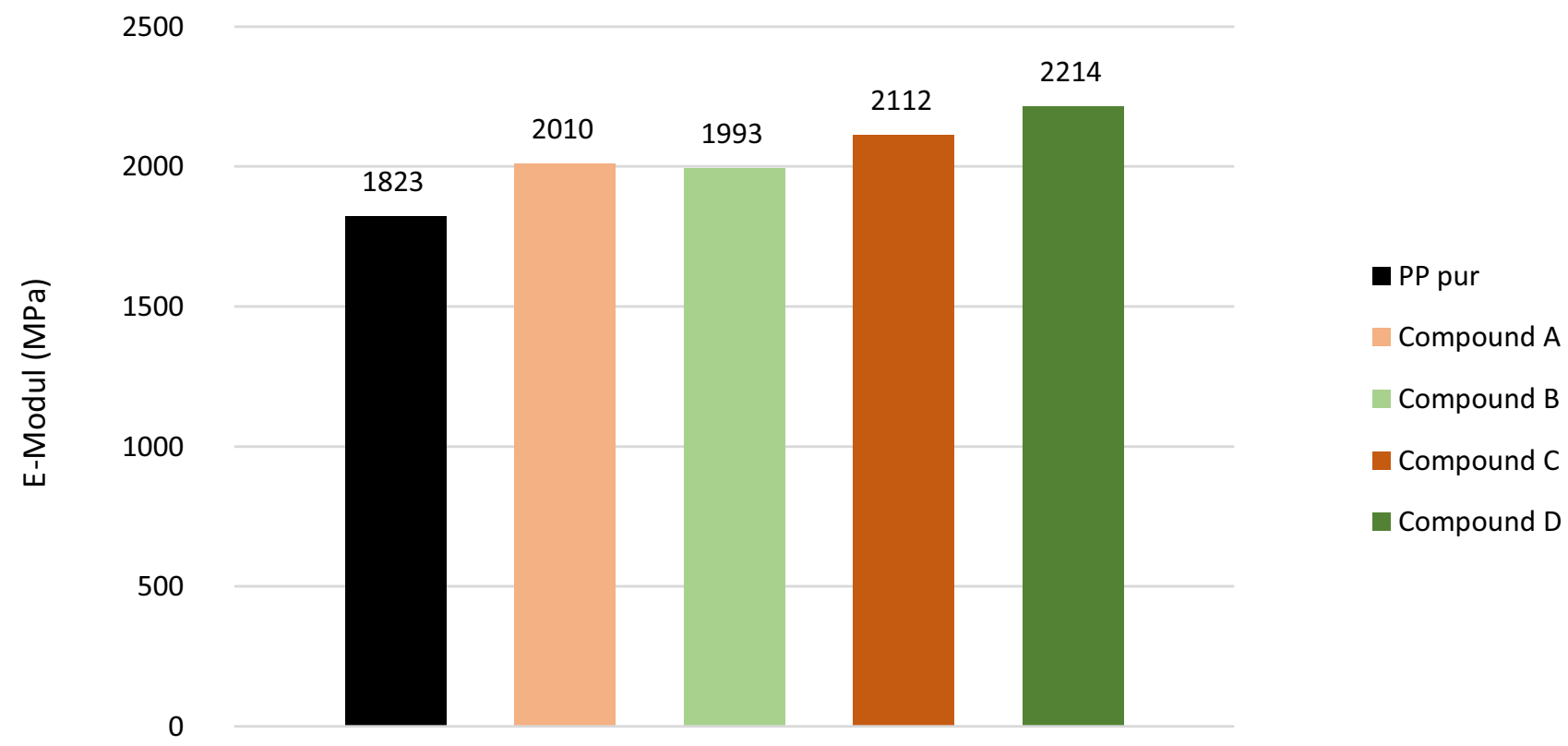

Vergleich E-Moduln der unterschiedlichen Compounds

Abb. 14: Vergleich des E-Moduls bei unterschiedlichen Füllgraden mit und ohne Verwendung eines Compatibilizers. Legende: Compound A: $95 \%$ PP Bormed ${ }^{\mathrm{TM}}$ DM55pharm $+5 \%$ Bentonit;

Compound B: $90 \%$ PP Bormed ${ }^{\mathrm{TM}}$ DM55pharm +5\% Compatibilizer Scona TPPP $2112+5 \%$ Bentonit;

Compound C: $85 \%$ PP Bormed $^{\mathrm{TM}}$ DM55pharm +15\% Bentonit;

Compound D: $80 \%$ PP Bormed $^{\mathrm{TM}}$ DM55pharm +5\% Compatibilizer Scona TPPP $2112+15 \%$ Bentonit.

Quelle: A. Neunhäuserer, Lehrstuhl für Spritzgießen der Kunststoffe, Montanuniversität Leoben, 2019 
Steigerung des E-Moduls erzielt werden konnte. Dies trifft besonders auf den auch in der Literatur immer wieder zitierten Füllgrad von $5 \mathrm{~m} \%$ zu. Die Verteilung des Füllstoffes in "Compound $A^{\prime \prime}$ ist in Abb. 4 gezeigt.
3.2.2 Untersuchungen des Kunststoff-Institutes für die mittelständische Wirtschaft NRW $\mathrm{GmbH}$

Die mit dem Planetwalzenextruder erhaltenen Compounds wurden am Kunststoff-Institut für die mittelständische Wirt-
Abb. 15: Vergleich des E-Moduls bei unterschiedlichen Füllgraden ohne Verwendung eines Compatibilizers.

Quelle: Kunststoff-Institut für die mittelständische Wirtschaft NRW GmbH, D-Lüdenscheid

Abb. 16: Vergleich der Zugfestigkeiten bei unterschiedlichen Füllgraden ohne Verwendung eines Compatibilizers.

Quelle: Kunststoff-Institut für die mittelständische Wirtschaft NRW GmbH, D-Lüdenscheid
Abb. 17: Erzielte Steigerungsraten des E-Moduls in Abhängigkeit vom Füllgrad, erstellt aus Daten des Kunststoff-Institutes für die mittelständische Wirtschaft NRW GmbH, D-Lüdenscheid

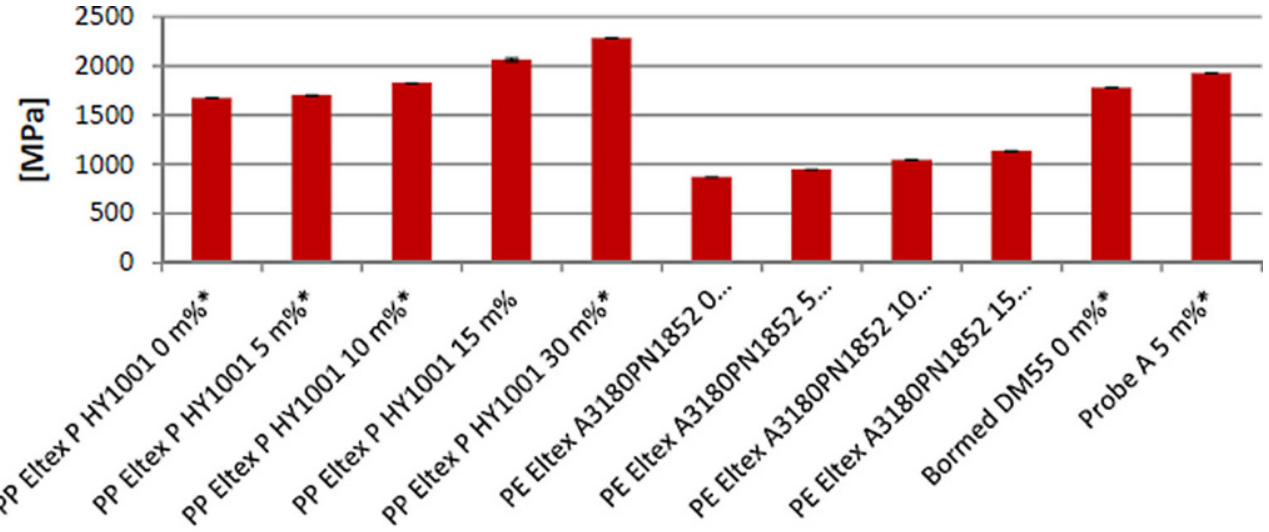

Kennwerte ermittelt nach DIN EN ISO 527

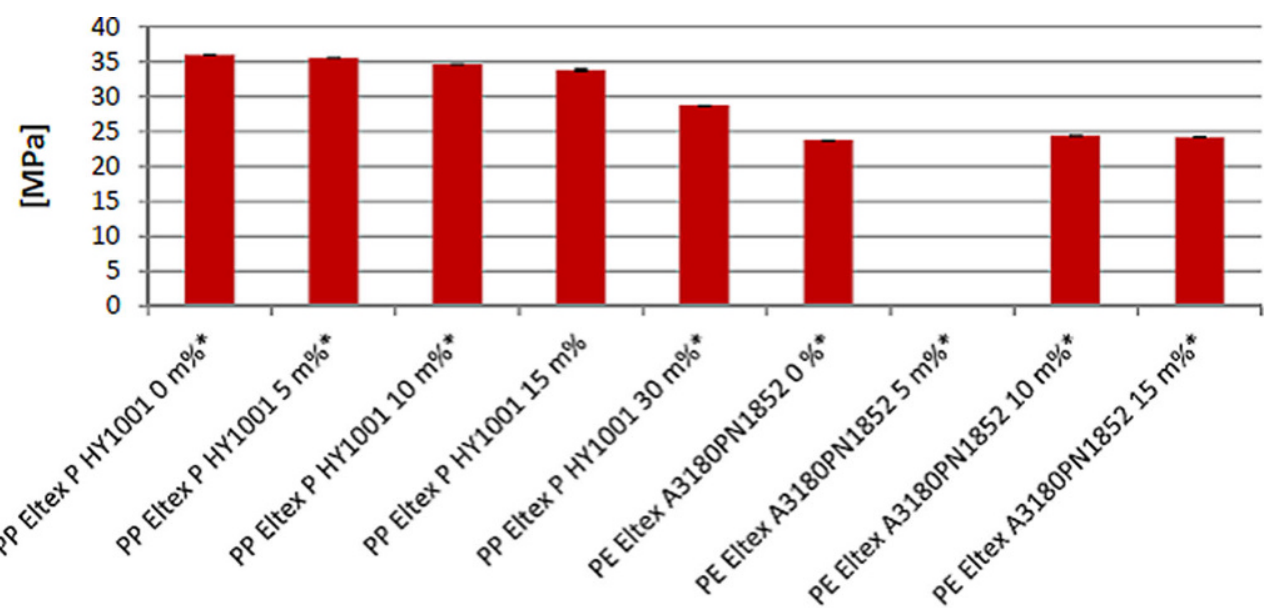

Kennwerte ermittelt nach DIN EN ISO 527

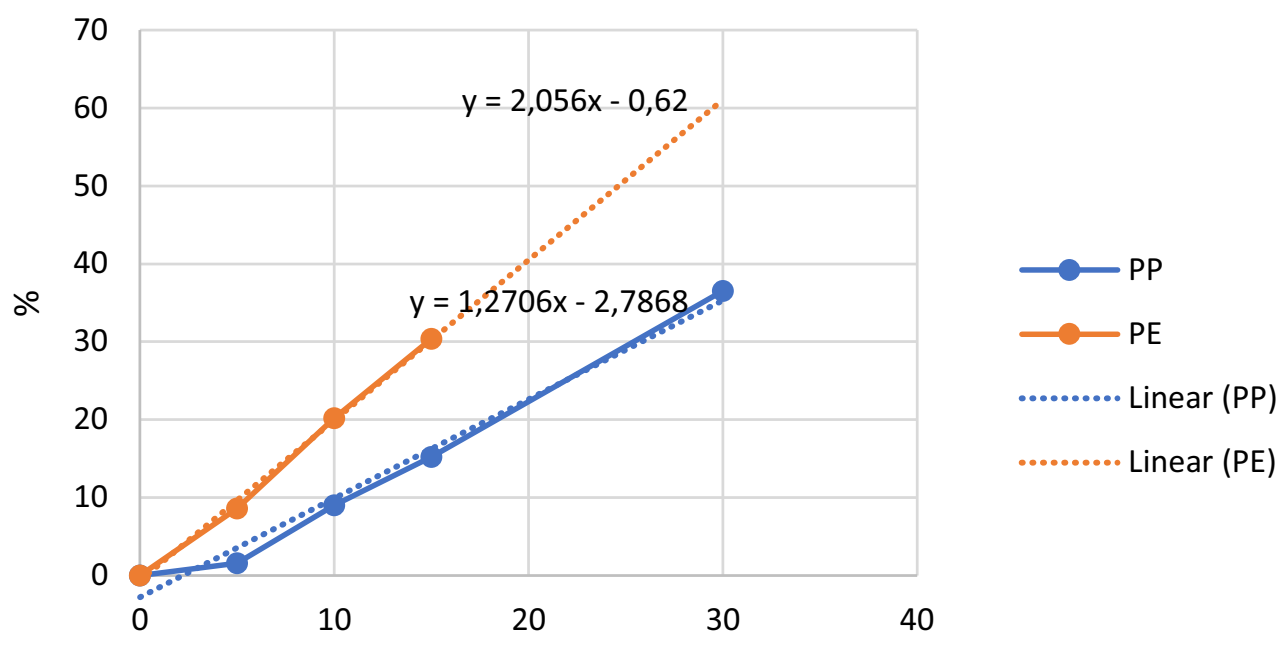

Füllgrad (\%) 
schaft NRW GmbH, Lüdenscheid, untersucht. Die nachfolgenden Darstellungen sind dem Prüfbericht NM 20.01331 vom 02.10.2020 entnommen (Abb. 15 und 16). Festgehalten wird, dass abweichend von der Norm lediglich ein Probestab geprüft wurde.

Bei Analyse der erzielten Ergebnisse ist klar festzustellen, dass die erreichten Absolutwerte sowohl beim E-Modul als auch bei den Zugfestigkeiten weit hinter den Erwartungen zurück blieben.

Dies trifft besonders bei dem verwendeten Polypropylen zu. Dafür scheinen zwei Gründe verantwortlich: Die erwartete Exfolierung wurde auch bei diesen ersten Versuchen mit dem Planetwalzenextruder noch nicht erreicht. Eine ausreichende Kompatibilität mit dem unstabilisierten homopolymeren Polypropylen ist offensichtlich nicht vorhanden.

Zwar führt die aus Abb. 11 und 12 erkennbare deutliche Verbesserung der Exfolierung beim Polyethylen auch zu einer Verdoppelung der Steigerungsraten des E-Moduls gegenüber Polypropylen, siehe Abb. 17, allerdings weisen die Zugfestigkeiten beim verwendeten Polypropylen ab einem Füllgrad von $15 \mathrm{~m} \%$ eine signifikant sinkende Tendenz auf. Die Ursache dafür erscheint eindeutig: Die nicht exfolierten Agglomerate wirken wie Fehlstellen in der Matrix des Verbundwerkstoffes. Trotz deutlich verbesserter Exfolierung und visuell nicht mehr erkennbarer Agglomerate bleibt die Zugfestigkeit beim Polyethylen im Bereich des Füllgrades bis $15 \mathrm{~m} \%$ praktisch konstant.

\section{Zusammenfassung}

Das erste Teilziel des Forschungsvorhabens, einen organophil modifizierten und damit geeigneten Nanoclay-Füllstoff auf Basis europäischer Kalzium-Bentonite herzustellen, konnte erreicht werden.

Das zweite, für eine industrielle Verwertbarkeit des Forschungsergebnisses gleichbedeutende Teilziel, die Einarbeitung in Polypropylen, ist auch mit diesen Versuchen verfehlt worden.

Im erreichten Stadium der Kompatibilität zu Polyolefinen und der Exfolierung erfüllt der organophil modifizierte Bentonit in seinem Verhalten nicht die Eigenschaften eines Verstärkungsstoffes.

Als weitere Schritte werden

- die elektronenmikroskopische Auswertung der Einbettung des Füllstoffes,

- die Klärung, warum es bei der Einarbeitung in Polypropylen zu einer extremen, und auch unter höheren Scherkräften nicht mehr auflösbaren Re-Agglomerierung auf das fast 60-fache der ursprünglichen Partikelgröße der vollständig hydrophoben MontmorillonitLamellen kommt,

- die Auswahl des für diesen Füllstoff geeigneten Polymers und gegebenenfalls Compatibilizers und

- die Fortsetzung der Versuche mit einem optimierten Planetwalzenextruder
Danksagung. Es ist den Autoren ein ausdrückliches Bedürfnis, folgenden Personen bzw. Institutionen besonderen Dank auszusprechen, ohne deren wertvolle und wohlwollende Unterstützung die Fortsetzung der Forschungsaktivitäten nicht möglich gewesen wäre. Namentlich dankt das Autorenteam (die Aufzählung erfolgt in der Chronologie der Arbeiten) Herrn E. Streibl, MSc., PMT Powde Processing GmbH, Kammern (A), Herrn B. Delbach, JM Polymers, Bochum (D), Herrn B. Schöneberg, Biesterfeld Plastic GmbH (D), Herrn Dipl.-Ing. K. Wallis, BKFMaschinentechnik, Bochum (D), den Herren Dipl.-Ing. B. Haberman und Dipl.Ing. M. Waffenschmidt, beide ENTEX Rust\&Mitschke GmbH, Bochum (D), Herrn Dr. W. Öfner, Lehrstuhl für Aufbereitung und Veredlung, Montanuniversität Leoben (A) und den Herren Dipl.-Ing. M. Tesch, Dipl.-Ing. D. Caparole und Dipl.-Ing. I. Eroglu, alle Kunststoff-Institut für die mittelständische Wirtschaft NRW GmbH Lüdenscheid (D).

Funding. Open access funding provided by Montanuniversität Leoben.

Open Access Dieser Artikel wird unter der Creative Commons Namensnennung 4.0 International Lizenz veröffentlicht, welche die Nutzung, Vervielfältigung, Bearbeitung, Verbreitung und Wiedergabe in jeglichem Medium und Format erlaubt, sofern Sie den/die ursprünglichen Autor(en) und die Quelle ordnungsgemäß nennen, einen Link zur Creative Commons Lizenz beifügen und angeben, ob Änderungen vorgenommen wurden.

Die in diesem Artikel enthaltenen Bilder und sonstiges Drittmaterial unterliegen ebenfalls der genannten Creative Commons Lizenz, sofern sich aus der Abbildungslegende nichts anderes ergibt. Sofern das betreffende Material nicht unter der genannten Creative Commons Lizenz steht und die betreffende Handlung nicht nach gesetzlichen Vorschriften erlaub ist, ist für die oben aufgeführten Weiterverwendungen des Materials die Einwilligung des jeweiligen Rechteinhabers einzuholen.

Weitere Details zur Lizenz entnehmen Sie bitte der Lizenzinformation auf http://creativecommons.org/licenses/by/4.0/deed.de.

\section{Literatur}

1. Le Baron, P.C.; Wang, Z.; Pinnavaia, T.J.: Polymer-layered silicate nanocomposites: an overview, Applied Clay Science, 15 (1999), iss. 1-2, pp 11-29. https://doi.org/10.1016/S0169-1317(99)00017-4

2. Zakaria, Z: Distribution of Nanoclay Particles in Polymer Composites,Universiti Teknologi Mara (UitM) 2018. https://www.youtoube. com/watch?v=gr_sBZYSVQ „Individual Assignment BCT 598 (Nanocomposits Technology)"

3. Witschnigg, A.; Laske, S.; Kracalik, M., Holzer, C.: Influence of Induced Shear Work on the Properties of Polyolefine Nanocomposite Pipes, Polymer Engineering \& Science, 52 (2012), iss. 5, https://doi. org/10.1002/pen.22146

4. Technisches Datenblatt BYK "Cloisite $20 \mathrm{~A}^{\prime \prime}$

5. Krischey, E.; Ohrdorf, K.-H.; Flachberger, H.: Verfahren zur Herstellung von alkalisch und organophil aktiviertem Kalzium-Montmorillonit für den Einsatz in Polymer-Compounds, BHM Berg- und Hüttenmännische Monatshefte 160 (2015), H. 6, S. 291-299

6. Ohrdorf, K.-H.: Zu Fragen der alkalischen Aktivierungvon Bentoniten, Dissertation Montanuniversität Leoben, 2010

7. Krischey, E.: Verfahren zur Herstellung von alkalisch und organophil aktiviertem Kalzium-Montmorillonit für den Einsatz in PolymerCompounds, Dissertation Montanuniversität Leoben, 2015

Hinweis des Verlags. Der Verlag bleibt in Hinblick auf geografische Zuordnungen und Gebietsbezeichnungen in veröffentlichten Karten und Institutsadressen neutral.

ins Auge gefasst. 